\title{
Cardio-renal syndrome.
}

\author{
Dhiraj Kumar*, Abhijeet Yelale, Girish Sabnis, Hetan Shah, Charan Lanjewar, Prafulla Kerkar \\ King Edward Memorial Hospital, Mumbai, Maharashtra, India
}

\begin{abstract}
Although newer pharmacologic and interventional therapies for the treatment of Heart failure continue to evolve, the optimum strategy of management for the patients with cardio renal syndrome is still unanswered. Various pathophysiologic mechanisms have been proposed including the low output hypothesis and the raised renal venous pressure hypothesis. These mechanisms have been tested and challenged in many trials. Although, modest benefit has been achieved with management strategies targeting these hypotheses. Role of biomolecules and neurohormones such as BNP, adenosine has provided newer insights in mechanisms of Cardiorenal syndrome (CRS). However, when studied in clinical trials, drugs targeting these pathways did not have significant clinical outcomes. Diuretics remain cornerstone of management. But their propensity to cause AKI and electrolyte disturbances when used at higher dosages limits their use. Vasodilator proves of clinical benefit in raised CVP states. But risk of hypotension and constant hemodynamic monitoring preclude their use in many clinical situations. Ultrafiltration had modest success in the initial studies, but recent trials have questioned their role in patients with ADHF. Pharmacologic therapies such as nesiritide, adenosine antagonists and vasopressin antagonists are still in trial phases and benefit has not yet been proved. Thus, our knowledge of CRS still needs depth and understanding of pathophysiologic mechanisms which when achieved may result in improved clinical outcomes and patient care. This review article on focuses on pathophysiology and management strategies of patients with CRS.
\end{abstract}

Keywords: Cardio renal syndrome, Heart failure, RAAS.

Accepted on April 16, 2018

\section{Introduction}

Understanding the interaction between heart and the kidneys has been the age-old question in the practice of medicine. Many theories have been postulated to solve this enigmatic problem and few have supportive evidences. Since decades, it has been known that the presence of renal dysfunction in settings of heart failure purports poor prognosis. Hence, over these years there has been a continuous effort to unfold the various mechanisms behind the complex interplay of heart and renal axis.

\section{Definition and Classification}

In 2004, the NHLB Institute tried in defining the CRS. It stated CRS a condition in which therapy to relieve congestive symptoms of HF is limited by a decline in renal function as manifested by a reduction in GFR [1]. Cardio renal syndrome (CRS) is an umbrella term that defines disorders of the heart and kidneys whereby "acute or chronic dysfunction in one organ may induce acute or chronic dysfunction of the other organ. Worsening renal function is most commonly defined as an absolute increase in serum creatinine of $\geq 0.3 \mathrm{mg} / \mathrm{dl} \mathrm{[2].}$

In 2010, at a consensus conference of the Acute Dialysis Quality Initiative (ADQI), the CRS was classified into five subtypes primarily based upon the organ that initiated the insult as well as the acuity of disease (Table 1) [3].

Many others including Braam et al. [4] had their individual opinions regarding this classification. They said this classification based on a time frame is too simplistic. They viewed CRS in a more integrated manner. They defined the cardio renal syndrome as a pathophysiological condition in which combined heart and kidney dysfunction amplifies progression of failure of the individual organ, by inducing similar pathophysiological mechanisms. Therefore, regardless of which organ fails first, the same neurohormonal systems are activated causing accelerated cardiovascular disease, and progression of damage and failure of both organs. These systems are broken down into two broad categories of "hemodynamic factors" and non-hemodynamic factors or "cardio renal connectors".

\section{Problem Statement}

Presence of renal dysfunction in heart failure has reported in numerous studies. An overall prevalence of $30-60 \%$ is mentioned [5]. In a study by Ezkowitz et al., [6] in 6,427 patients with cardiologist-diagnosed HF and angiographically proven coronary artery disease (mean age 69 years; 65\% men; one-year mortality, $10 \%$ ), $39 \%$ had creatinine clearances $<60$ $\mathrm{ml} / \mathrm{min}$. In the ADHERE study, The Acute Decompensated Heart Failure National Registry (ADHERE) database reported data on over 100,000 patients with HF requiring hospitalization, approximately 30 percent had a diagnosis of chronic kidney disease (defined as a serum creatinine greater than $2.0 \mathrm{mg}$ / dL. Over $60 \%$ of patients admitted in this registry with acute decompensated HF (ADHF) had stage 3 (GFR $<60 \mathrm{ml} / \mathrm{min}$ per $1.73 \mathrm{~m}^{2}$ ) or worse CKD. One study showed echo evidence of $\mathrm{LVH}$ in $45 \%$ patients with creatinine clearance of $<24 \mathrm{ml} / \mathrm{min}$ and in $70 \%$ of those planning to initiate haemodialysis [7]. The prevalence of CKD (type 2 CRS) is seen in $32 \%-50 \%$ of patients in the large chronic HF trials [7]. 
In the Valsartan in Heart Failure Trial [8] age, men, diabetes, ischemic aetiology of HF, low BPs, worse neurohormonal and proinflammatory profiles, presence of oedema, and use of higher doses of diuretics were independently associated with the presence of CKD (Table 2).

\section{Pathophysiology of CRS}

Many theories and hypothesis have been postulated to determine the exact mechanisms by which these two systems interact. The following commonly mentioned hypothesis include: -

\section{The Low flow state hypothesis}

It is based on our traditional understanding that a decreased cardiac output results in a decreased renal perfusion pressure indirectly affecting GFR. A severe decrease in LV function causes a reduction in the $\mathrm{CO}$ and threatens the arterial $\mathrm{BP}$, which lead to carotid sinus and aortic arch baroreceptor-mediated activation of several neurohormones. The parasympathetic tone is inhibited, and the sympathetic tone is enhanced, with subsequent activation of the RAAS.

However, this hypothesis had its caveats. The ESCAPE trial [9] evaluated the effectiveness of pulmonary artery catheterization in 433 patients with acute decompensated HF. There was no correlation between the cardiac index and either the baseline GFR or worsening kidney function and increasing the cardiac index did not improve renal function after discharge. Similar findings were noted in another report in which HF patients with worsening kidney function did not have lower cardiac outputs or filling pressures than those without worsening kidney function.

\section{Raised renal venous pressure}

Estimation of CVP and its role in managing patients with heart failure is of prime importance. Patients with heart failure have raised CVP which also increases renal venous pressure there by decreasing the gradients across glomerular capillary network resulting in reduced GFR. Increased intra-abdominal pressure causes renal venous pressure to rise. Trans-renal perfusion pressure is calculated as mean arterial pressure minus the central venous pressure. Therefore, for the patient with volume overload and heart failure, the combination of increased pulmonary artery or central venous pressure with low systemic pressure may lead to a severe compromise of the net renal perfusion pressure.

Therefore, whenever there is an opportunity to reduce the central venous pressure, whether through vasodilation, improved oxygenation or volume reduction, this can result in significant improvements in renal blood flow and urine output. Many animal and human studies have demonstrated a concomitant decrease in GFR as intraabdominal pressure rises. The concept that venous congestion, not arterial blood flow, is an important mediator of cardio renal failure is supported by the findings of the Evaluation Study of Congestive Heart Failure and Pulmonary Artery Catheterization Effectiveness trial, in which only baseline right atrial pressure, not arterial blood flow, correlated with baseline serum creatinine (Figure 1).

\section{Neurohormonal Mechanisms}

In a patient in heart failure, many biochemical molecules are released in circulation, some of which prove to be of prime importance in the early course of the disease but end up being counterproductive. Best known among these are sympathetic system stimulation i.e. catecholamines. With reduction in stroke volume there is a surge of catecholamines in the blood stream. However, over the time, the cause renal vasoconstriction and reduction in GFR. These hormones are in fact responsible for ventricular remodelling.

Decreased systolic function is detrimental to the kidneys via the activation of the RAAS system. Angiotensin II not only has its effects in the kidneys, but also in other organ systems to contribute to hypertension, $\mathrm{LV}$ remodelling and $\mathrm{Na}$ and Volume retention. AT-II exerts many deleterious effects through the activation of NADPH oxidase and NADH oxidase. AT-II activates these 2 enzymes within vascular smooth muscle cells, cardiac myocytes, and renal tubular epithelial cells, generating superoxide, a reactive oxygen species.

Oxidative stress is a hallmark of type $1 \mathrm{CRS}$, as evidenced

Table 1. Types of Cardio renal syndrome.

\begin{tabular}{|c|c|c|c|}
\hline Type & Inciting event & Secondary disturbance & Example \\
\hline Type 1 (acute CRS) & $\begin{array}{l}\text { Abrupt worsening of heart } \\
\text { function }\end{array}$ & kidney injury & $\begin{array}{l}\text { acute cardiogenic shock or acute decompensation } \\
\text { of chronic heart failure }\end{array}$ \\
\hline Type 2 (chronic CRS) & $\begin{array}{l}\text { Chronic abnormalities in } \\
\text { heart function }\end{array}$ & progressive chronic kidney disease & chronic heart failure \\
\hline $\begin{array}{c}\text { Type } 3 \text { (acute Reno cardiac } \\
\text { syndrome) }\end{array}$ & $\begin{array}{c}\text { Abrupt worsening of kidney } \\
\text { function }\end{array}$ & $\begin{array}{l}\text { acute cardiac disorder (e.g. heart failure, abnormal heart } \\
\text { rhythm, or pulmonary oedema) }\end{array}$ & acute kidney failure or glomerulonephritis \\
\hline $\begin{array}{l}\text { Type } 4 \text { (chronic Reno } \\
\text { cardiac syndrome) }\end{array}$ & Chronic kidney disease & $\begin{array}{l}\text { decreased cardiac function, cardiac hypertrophy and/or } \\
\text { increased risk of adverse cardiovascular events }\end{array}$ & chronic glomerular disease \\
\hline Type 5 (secondary CRS) & Systemic condition & both heart and kidney dysfunction & diabetes mellitus, sepsis, lupus \\
\hline
\end{tabular}

Table 2. Common risk factors for CRS.

\author{
-Older age \\ Comorbid conditions (diabetes mellitus, uncontrolled hypertension, anaemia) \\ -Drugs (anti-inflammatory agents, diuretics, ACE inhibitors, ARBs) \\ -History of heart failure or impaired left ventricular ejection fraction \\ -Prior myocardial infarction \\ -New York Heart Association (NYHA) functional class \\ -Elevated cardiac troponins \\ -Chronic kidney disease (reduced Egfr, elevated BUN, creatinine, or cystanin)
}




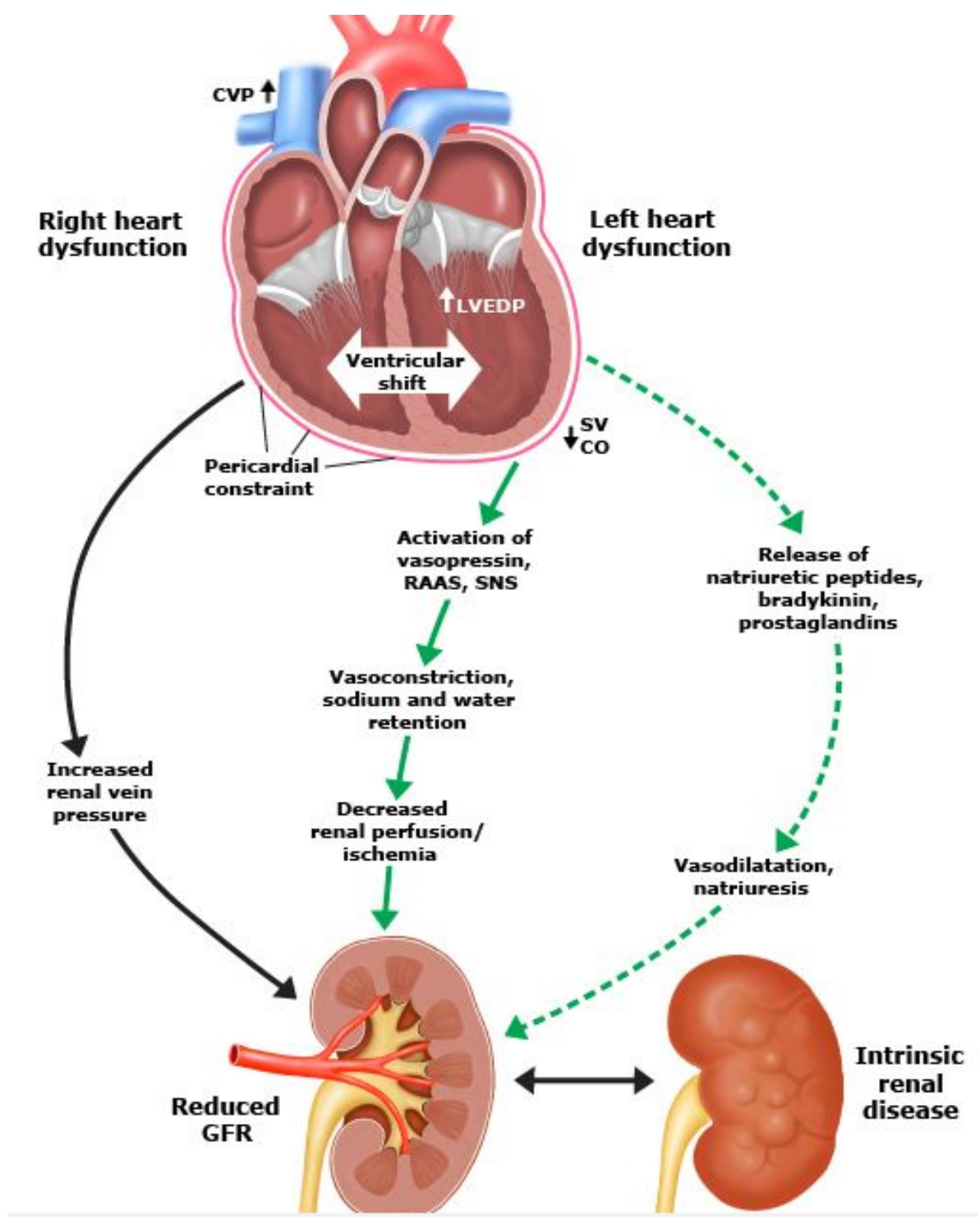

Figure 1. Schematic diagram depicting interaction between the heart and kidney.

by a significant increase in circulating reactive oxygen species (ROS) and reactive nitrogen species (RNS), coupled with increased expression of interleukin-6 (IL-6). Increased levels of NADPH oxidase and myeloperoxidase (MPO), with upregulation of proinflammatory mediators via powerful oxidants such as peroxynitrite. Neuro-hormonal activation, renal hypoperfusion and venous congestion, inflammation, atherosclerosis and oxidative stress represent most important pathophysiological mechanisms of type $2 \mathrm{CRS}$ also (Figure 2) [10].

\section{Adenosine}

A recently identified contributing factor is adenosine and the related tubuloglomerular feedback. Adenosine can be locally released in the kidney under stress and binds to receptors on the afferent arterioles and cause vasoconstriction, thereby reducing renal blood flow. Stimulation of the receptor also increases sodium resorption in the tubules, leading to further sodium and water retention. Acute delivery of sodium to the distal tubules by diuretic therapy in acute decompensated heart failure will in turn stimulate further adenosine release from macula densa, and further reduce glomerular filtration.

Besides these, Vasopressin levels are found to be elevated in patients with heart failure. Vasopressin causes increased absorption of water in DCT and results in volume retention. However, the clinical benefits of vasopressin antagonists have not been proven in patients with heart failure though they cause modest decrease in volume status.

\section{Role of Medications}

With diuretics being the cornerstone of management of patients in acute heart failure, there are many factors that limit their usage. The foremost and one of the most common problems in ICCU being over diuresis leading increase in creatinine levels and reduction in GFR. It is important to recognize and differentiate this problem other causes of AKI for optimal management of heart failure.

Furosemide can increase fibrosis by its known stimulation of the renin-angiotensin-aldosterone axis (Figure 3). Metabolic alkalosis is another frequent problem which limits diuretic usage. 


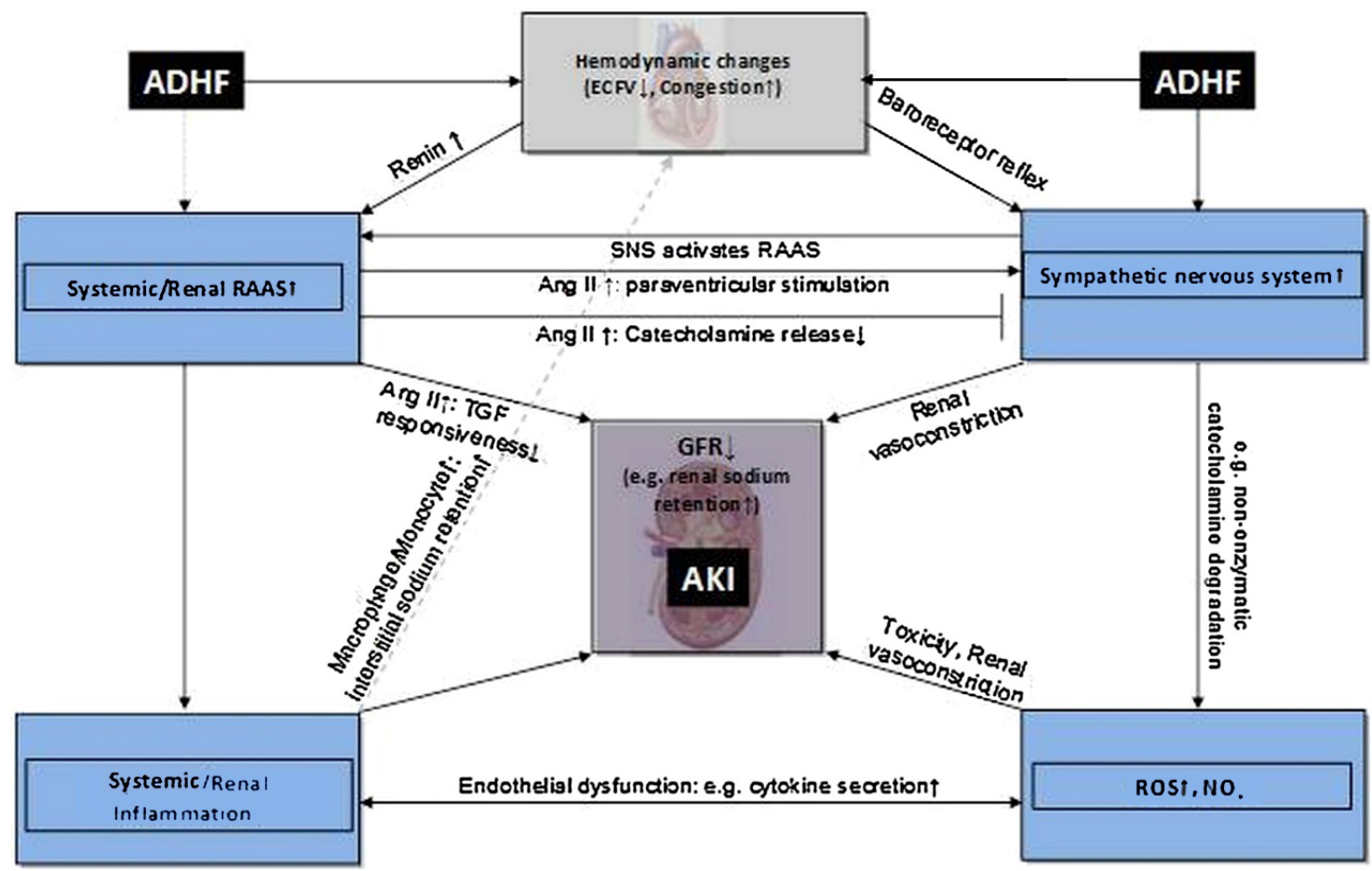

Figure 2. Role of neuro-humoral systems in CRS.

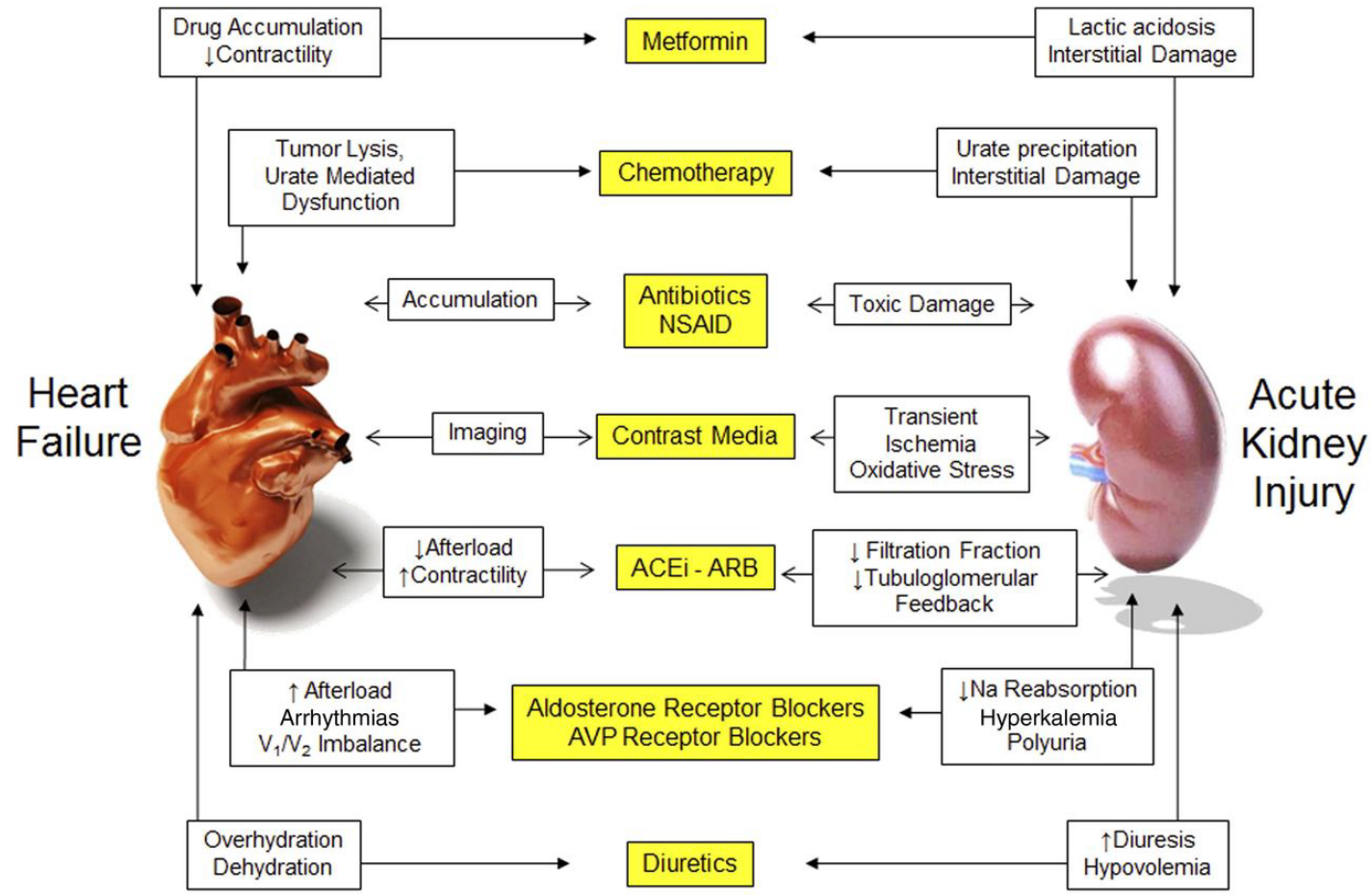

Figure 3. How common medications impact the heart kidney axis.

ACE Inhibitors and ARBs have now become a standard of care in heart failure patients. Although the beneficial effects are par, it must be emphasised that they cause a transient rise in creatinine levels due the efferent arteriolar vasodilation (which results in decrease in intraglomerular filtration pressure). Hence, cautious use is recommended in those patients who already have reduced renal reserve; the main risk being hyperkalaemia.

\section{Diagnosis}

Whereas the diagnosis of type 1 and type 2 cardiorenal syndromes essentially requires estimation of GFR, diagnosing CRS in patients with primary renal involvement (type 3 and type 4) requires clinical suspicion as well as appropriate investigations. Many patients with CKD have accelerated atherosclerosis leading to CAD. It is important that such patients complaining of fatigue and dyspnoea, despite optimum medical therapy might have reduced ejection fraction and CAD. These patient warrant evaluations by echocardiography and if required Coronary angiography. More than half of CKD patients have hypertensive heart disease as proven on echo. Such patients are prone to develop HefPef. 


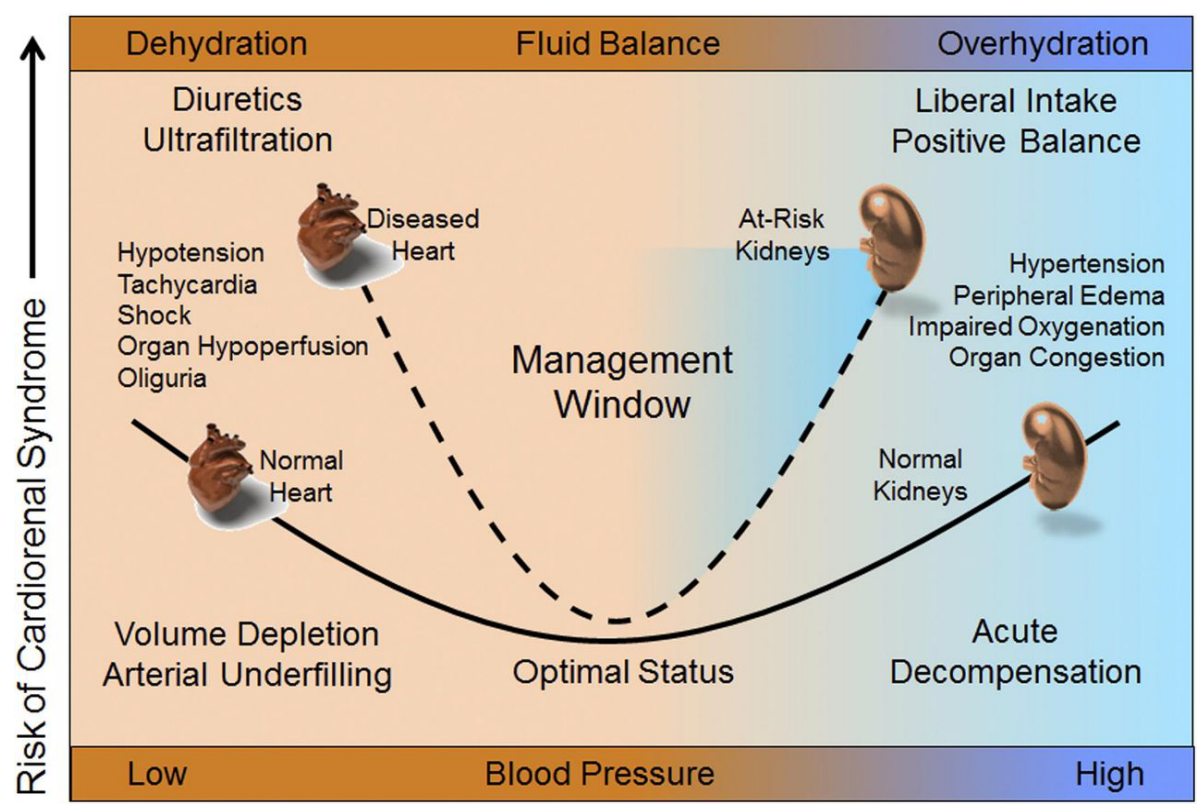

Figure 4. Concept of fluid status in understanding of CRS.

Measurement of the urine sodium concentration also may be helpful. A urine sodium concentration below $25 \mathrm{meq} / \mathrm{L}$ would be expected with HF, since renal perfusion is reduced with associated activation of the renin-angiotensin-aldosterone and sympathetic nervous systems, both of which promote sodium retention. However, higher values may be seen with concurrent diuretic therapy if the measurement is made while the diuretic is still acting.

\section{Management}

Among patients with HF who have an elevated serum creatinine and/or a reduced estimated GFR, it is important to distinguish between underlying kidney disease and impaired kidney function secondary to the cardiac complication. This distinction may be difficult, and some patients have both underlying chronic kidney disease and CRS.

Optimising Heart Failure medications is of utmost importance.

The following Canadian Cardiovascular Society guidelines help for an early diagnosis as well as prevention of CRS (Table 3) [11].

\section{Diuretics}

Loop diuretics can be of benefit in patients predominantly with right heart failure and AKI. They decrease renal venous pressure by decongestion. However, over diuresis should be avoided.

The effect of diuretic-induced fluid removal on the glomerular filtration rate is quite variable in patients with HF (Figure 4):

- With the onset of diuretic therapy, there occurs a decrease in cardiac filling pressures which results in a decrease in cardiac output. This decrease in cardiac output can lead to decrease in renal perfusion pressures and hence an increase in creatinine levels [12].

- Some patients lie on flat part of Frank-Starling curve. Hence there is no change in creatine levels. On this part of the curve, a decrease in filling pressures has negligible impact on Cardiac output.

- Few patients end up with decrease in creatinine levels which could be due to reduction in intraabdominal and central venous pressures.

In spite all these possibilities, diuretics remain the cornerstone of the management of patients of acute heart failure. A transient or mild increase in creatinine should not refrain physicians from using diuretics.

Diuretic resistance is another problem which poses challenge in management of patients with CRS. In the setting of ADHF, there is gut oedema and rise in intra-abdominal pressure which leads to decrease in oral absorption of loop diuretics. Hence IV Furosemide is used routinely in ADHF patients. However, even with IV diuretics, there is risk of rebound increase in $\mathrm{Na}$ reabsorption. Addition of metolazone in patients with diuretic resistance might help in modest increase in increasing the urine output.

\section{Vasodilators}

IV Nitro-glycerine, a potent vasodilator has a role in patients with CRF if used in right circumstances. Frequent dose titration may be necessary to avoid hypotension. NTG improves stroke volume by decreasing the SVR. It also decreases the CVP by venodilation and thus decreases renal perfusion pressure.

Recombinant human B-type natriuretic peptide, or nesiritide, is an effective vasodilator with mild diuretic effects. However, the results from ASCEND-HF trial showed that nesiritide does not have any role in management of patients with heart failure. Rather, it has worse renal outcome [13].

\section{Ultrafiltration}

It was hypothesized that removing the excess volume in patients with CRS might prove beneficial. Ultrafiltration involves the removal of plasma water across a semipermeable membrane 
Table 3. Canadian cardiovascular society consensus recommendations on heart failure and concomitant renal dysfunction.

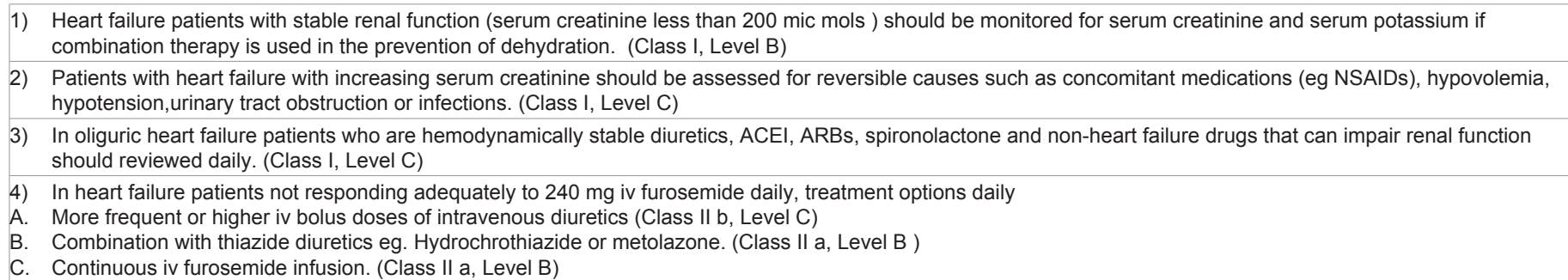

in response to a transmembrane pressure gradient. There is no evidence favouring ultrafiltration over loop diuretics as firstline therapy in patients with AHF. At present, routine use of ultrafiltration is not recommended and should be confined to patients who fail to respond to diuretic based strategies.

Three randomized trials (UNLOAD, RAPID-CHF, and CARESS-HF) [14-16] compared ultrafiltration to diuretic therapy in patients with acute decompensated HF. The mean baseline serum creatinine levels were $1.5,1.7$, and $2.0 \mathrm{mg} /$ $\mathrm{dL}$, respectively. In UNLOAD and RAPID-CHF, ultrafiltration was associated with a significantly greater rate of fluid loss than diuretic therapy but no difference in serum creatinine. In CARESS-HF, ultrafiltration was compared to stepped pharmacologic therapy (including bolus plus high doses of continuous infusion loop diuretics, addition of thiazide diuretic (metolazone ), and selected intravenous inotrope and/ or vasodilator therapy) in patients with worsening renal function and persistent congestion. Although weight loss was similar in ultrafiltration and stepped pharmacologic therapy groups, ultrafiltration therapy caused an increase in serum creatinine and a higher rate of adverse events.

\section{Inotropes}

Dobutamine, dopamine and milrinone are used mainly patients with cardiogenic shock. Even though these drugs have been shown to increase mortality, they continue to be used in primarily in patients with cardiogenic shock and selected patients with ADHF. The role of inotropes in patients with CRS is uncertain and trials are underway to better define their role. At present, the routine use of inotropes cannot be recommended given their association with adverse events when used outside of selected patients with cardiogenic shock or acute decompensated HF.

\section{Conclusion}

Even though our knowledge of understanding the cardio-renal axis is increasing, much research work and investigational newer therapies are needed for the optimum management of $\mathrm{C}$.

\section{References}

1. NHLBI Working Group. Cardio-renal connections in heart failure and cardiovascular disease. National Heart, Lung, and Blood Institute Web site.

2. Cowie MR, Komajda M, Murray-Thomas $T$, et al. Prevalence and impact of worsening renal function in patients hospitalized with decompensated heart failure: results of the Prospective Outcomes Study in Heart Failure (POSH). Eur Heart J. 2006;27:1216-22.
3. Ronco C, McCullough SD. "Cardio-renal syndromes: Reports from the consensus conference of the acute dialysis quality initiative". Eur Heart J. 2010;31:703-11.

4. Braam Branko, Joles Jaap A, Danishwar Amir H, et al. "Cardiorenal syndrome-current understanding and future perspectives". Nature Reviews Nephrology. 2013;10:48-55.

5. Adams KF Jr, Fonarow GC, Emerman CL, et al. Characteristics and outcomes of patients hospitalized for heart failure in the United States: rationale, design, and preliminary observations from the first 100,000 cases in the Acute Decompensated Heart Failure National Registry (ADHERE). Am Heart J. 2005;149:209-16.

6. McAlister FA, Ezekowtiz J, Tonelli M, et al. Renal insufficiency and heart failure: prognostic and therapeutic implications from a prospective cohort study. Circulation. 2003;109:1004-9.

7. Parfrey PS, Foley RN, Harnett JD, et al. Outcome and risk factors for left ventricular disorders in chronic uraemia. Nephrol Dial Transplant.1996;11:1277-85.

8. Jay N Cohn, Gianni Tognoni. Randomized Trial of the Angiotensin-Receptor Blocker Valsartan in Chronic Heart Failure December 6, 2001N Engl J Med. 2001;345:1667-75.

9. Nohria A, Hasselblad V, Stebbins A, et al. Cardiorenal interactions: insights from the ESCAPE trial. J Am Coll Cardiol. 2008;51:1268.

10. Virzì GM, Clementi A, DeCal M, et al. Oxydative stress: dual pathway induction in cardiorenal syndrome type 1 pathogenesis. Oxidative Medicine and Cellular Longevity. 2015;1-9.

11. Canadian Cardiovascular Society consensus conference recommendations on heart failure 2006: diagnosis and management. Can J Cardiol. 2006;22:23-45.

12. Stampfer M, Epstein SE, Beiser GD, et al. Hemodynamic effects of diuresis at rest and during intense upright exercise in patients with impaired cardiac function. Circulation. 1968;37:900.

13. O'Connor CM, Starling RC, Hernandez AF, et al. Effect of Nesiritide in Patients with Acute Decompensated Heart Failure. N Engl J Med. 2011;365:32-43.

14. Costanzo MR, Guglin ME, Saltzberg MT, et al. Ultrafiltration versus intravenous diuretics for patients hospitalized for acute decompensated heart failure. J Am Coll Cardiol. 2007;49:675. 
15. Bart BA, Boyle A, Bank AJ, et al. Ultrafiltration versus usual care for hospitalized patients with heart failure: the Relief for Acutely Fluid-Overloaded Patients with Decompensated Congestive Heart Failure (RAPID-CHF) trial. J Am Coll Cardiol. 2005;46;2043.
16. Bart BA, Goldsmith SR, Lee KL, et al. Ultrafiltration in decompensated heart failure with cardiorenal syndrome. N Engl J Med. 2012;367:2296.

\section{*Correspondence to:}

Dhiraj Kumar

King Edward Memorial Hospital

Mumbai

Maharashtra

India

E-mail:dhiraj1645@gmail.com 